\title{
Germanica
}

\section{Réflexions allemandes sur l'idée de progrès et sur le rôle de la mémoire dans sa constitution, son évaluation et sa subversion}

Überlegungen über die Fortschrittsidee und die Rolle des Gedächtnisses

Niall BOND

\section{(2) OpenEdition}

Journals

Édition électronique

URL : http://journals.openedition.org/germanica/1834

DOI : 10.4000/germanica.1834

ISSN : 2107-0784

Éditeur

Université de Lille

Édition imprimée

Date de publication : 31 décembre 2003

Pagination : 159-182

ISBN : 9782913857124

ISSN : 0984-2632

Référence électronique

Niall BOND, «Réflexions allemandes sur l'idée de progrès et sur le rôle de la mémoire dans sa

constitution, son évaluation et sa subversion », Germanica [En ligne], 33 | 2003, mis en ligne le 03

décembre 2012, consulté le 06 octobre 2020. URL : http://journals.openedition.org/germanica/1834 ;

DOI : https://doi.org/10.4000/germanica.1834

Ce document a été généré automatiquement le 6 octobre 2020.

(c) Tous droits réservés 


\title{
Réflexions allemandes sur l'idée de progrès et sur le rôle de la mémoire dans sa constitution, son évaluation et sa subversion
}

\author{
Überlegungen über die Fortschrittsidee und die Rolle des Gedächtnisses
}

\author{
Niall BOND
}

Si l'on espère, en étudiant l'Histoire des idées politiques, sociales ou économiques en Allemagne, éclairer non seulement la teneur des idées ou la relation logique qui existe entre elles, mais également la place qu'elles occupent dans « la concaténation unique de circonstances » qu'est, d'après Max Weber, l'Histoire, les pièges d'une telle lecture sont multiples. Dans le contexte historique des études germaniques en France, Gérard Raulet nous met en garde contre les risques conjoints d'une réduction de la pensée à des «idées allemandes» et d'une "histoire culturelle politisée ». À l'époque de la République de Weimar déjà, on déplorait outre-Rhin la tendance des germanistes français à regrouper sous l'appellation de " pangermanisme... tout et n'importe quoi : le romantisme allemand, les aspirations allemandes à l'unité et le militarisme $»^{1}$. Nous traiterons ici différentes analyses des sens du concept de progrès élaborées par des auteurs allemands divers, tout d'abord sans "généalogie, ni téléologie »², et dans un souci de compréhension et non de relégation. Ces sources allemandes éclairent, souvent de manière exemplaire, le teneur du concept de progrès, les présupposés logiques qui le sous-tendent, ses rôles et ses récupérations politiques, économiques et sociales, ainsi que les conséquences psychologiques que l'intériorisation de cette idée peut entraîner. Elles aident à comprendre le rôle d'une reconstruction de la mémoire dans la destruction du progrès, et finalement à cerner le rapport nécessaire entre le progrès et la mémoire ${ }^{3}$. On ne saurait, en revanche, escamoter, purement et simplement, toute « histoire culturelle politisée » dans une discussion de l'Histoire des idées en Allemagne. 
2 Il s'agit ici, en effet, de comprendre aussi la réception de l'idée de progrès en Allemagne dans les interprétations politiques des auteurs allemands de l'après-guerre. Leur récit est celui d'un Sonderweg dont ils soulignent, avant tout, les périls ${ }^{4}$. La présente contribution esquissera également la place de l'idée de progrès dans la lecture libérale allemande d'un processus que le marxiste Lukács qualifia, après la chute du régime nazi, de « destruction de la raison $»^{5}$. À la suite de l'effondrement militaire et moral de l'Allemagne, les penseurs politiques de l'après-guerre - Plessner, von Krockow, Sontheimer, Fraenkel, Strauss, Hennis, Dahrendorf, Sternberger - ont tous, chacun à sa façon, vu la négation du " progrès » comme facteur majeur dans le rejet allemand du consensus des sociétés de l'Europe occidentale. L'histoire de cette idée en Allemagne au xxe siècle est aussi celle de la perception de son adoption et de son assimilation, de son utilisation et de sa récupération, mais devient surtout celle de son refus, de sa répudiation et de sa critique, face à l'essor de ce concept porteur d'espoirs politiques, économiques et sociaux dans les sociétés civiles voisines. L'échec du système rationnel et régulateur de la vie sociale fondé sur les institutions des «démocraties occidentales $\|^{6}$ mais sans ancrage dans une croyance aux vérités simples ${ }^{7} \mathrm{du}$ Droit naturel ${ }^{8}$ porteuses de stabilité ${ }^{9}$ 'est soldé par la " catastrophe allemande ». La critique allemande du progrès figure, chez bon nombre d'auteurs allemands de l'après-guerre, comme un chapitre de l'histoire intellectuelle du nazisme. On peut aisément voir que cette lecture limite, voire exclut une compréhension actuelle et rationnelle des idées allemandes ${ }^{10}$. Certaines réceptions de l'idée de progrès sont occultées; en corroborant l'hypothèse réductrice du Sonderweg, on commet les mêmes péchés épistémologiques que les évolutionnistes d'antan.

3 Plus généralement, le propre de l'Histoire étant de mettre en évidence les similitudes et les différences entre un objet et d'autres, toute Histoire est un Sonderweg. Il existe, outre-Manche - de l'économiste Smith au sociologue Spencer, en passant par l'historien Buckle - la conviction que la confiance dans les forces du « progrès » relevait d'un privilège insulaire; c'est grâce à la position géographique unique des îles britanniques que leur société représentait, pour Buckle, la référence pour toute évolution heureuse ${ }^{11}$. Refusant d'assimiler cette optique évolutionniste, certains auteurs de langue allemande se sont employés, au cours du dix-neuvième et au début du vingtième siècle, à décortiquer ces présupposés dans un contexte d'impérialismes concurrents, dans lequel le "progrès » fût défini par des auteurs français et anglosaxons en rapport avec les phénomènes spécifiques qui émergeaient au sein de leurs sociétés. Le projet de libérer l'Histoire allemande des jugements métaphysiques de l'Europe occidentale a été plus ou moins abandonné à la fin de la Deuxième Guerre mondiale, pour être partiellement repris plus tard sous de nouveaux auspices.

Cette écriture consistait, en partie, en une revalorisation du retard du développement des possibilités matérielles par rapport à d'autres sociétés européennes pendant l'ère de l'impérialisme européen. Le titre de l'ouvrage de Plessner, Die Verspätete Nation, est symptomatique de l'importance que l'on attribue, en Allemagne, aux décalages par rapport à d'autres nations européennes dans la centralisation, l'unification, la liberté d'échanges en tout genre et la mise en place de mécanismes pour assurer une représentation et un épanouissement de la société civile. Cette tendance resurgit dans la critique allemande des importations matérielles, symboliques et idéologiques depuis les balbutiements des sciences humaines chez certains auteurs caméralistes, qui rejettent très clairement la domination de pouvoirs mercantiles porteurs d'une 
idéologie de progrès teintée d'hédonisme et de tolérance, que ces auteurs interprètent, dès avant les Lumières, comme symptôme de déclin social ${ }^{12}$. (Les premiers retranchements disciplinaires derrière des frontières nationales remontent donc aux débuts d'une science de la défense des intérêts nationaux.)

5 L'intérêt réel de la critique du concept de progrès en Allemagne relève du fait que, pour un certain nombre d'auteurs allemands, notamment pendant l'ère wilhelminienne et la République de Weimar, l'idée de progrès n'était justement pas une «idée allemande ». L'affrontement actuel des civilisations révèle l'importance récurrente de lectures aliénées de l'idée occidentale de progrès - extension de la durée de l'existence humaine sous les auspices d'une amélioration constante du confort de l'individu - à une époque où l'Europe partage très largement les valeurs de cet hédonisme rationnel.

\section{Le progrès comme valeur scientifique et sociale}

6 Dans l'évaluation et l'auto-définition de toute société, l'idée de "progrès » (que l'on retrouve dans le langage du "développement»), était destinée à servir, depuis sa diffusion, de référence floue mais inéluctable à tout territoire et à ses habitants. Elle part de l'observation de différences dans les niveaux de confort matériel atteint par l'Homme dans son assujettissement du Monde par la rationalité instrumentale. La recherche des origines des richesses des nations est rapidement devenue le programme des sciences humaines dans le cadre institutionnel de l'État-nation ${ }^{13}$. Sans que la notion de progrès soit explicitée, le constat du progrès et de son retard dans différentes sociétés européennes est une impulsion fondamentale dans la genèse de l'ouvrage Richesse des Nations d'Adam Smith. L'idée de progrès est ensuite transposée de la réflexion technologique et économique à la réflexion sur la société; les projets de gestion envisagés par les utilitaristes et les premiers socialistes en Europe furent fondés sur une confiance accordée au progrès dans la production et la répartition matérielles ; les adeptes du saint-simonisme et les positivistes de toute obédience célébraient ce concept dans une vision quasiment religieuse. Les sciences humaines se destinaient, finalement, à l'instar des sciences naturelles, à retracer les grandes lignes et les chaînes causales de l'évolution sociale, "die ewig ehernen Gesetze des Sozialen", comme les qualifiera le père fondateur de la sociologie moderne allemande ${ }^{14}$, Ferdinand Tönnies, qui adopte les prétentions méthodologiques du positivisme, tout en se gardant d'intégrer ses biais idéologiques.

7 Si les fondateurs de la sociologie allemande semblent, dans leur ensemble, plus critiques par rapport au concept de progrès que les premiers grands sociologues français, c'est parce que leurs paradigmes fondamentaux ont été formulés en prenant en considération les conséquences de l'industrialisation, alors que la science positive française, dans laquelle cette idée a été aussi mobilisatrice, avait un siècle d'avance. La réception, les acceptions et les recupérations successives du concept de progrès en France, qui avait déjà obtenu un droit de cité privilégié dans les ouvrages de Condorcet et de Turgot ${ }^{15}$, témoignaient de son efficacité idéologique et de son utilité politique, tout comme de son ambivalence profonde. Si l'idée de progrès est, dans la vie politique, rapidement venue se substituer aux paroles légitimantes de l'Ancien régime - l'ouvrage de Turgot fut écrit en l'an trois de la Révolution -, son réel pouvoir social découlait des avancées technologiques et économiques. Le progrès, mot clef pour les acteurs de la société industrielle, décrivait d'abord le chemin vers une solution technique 
permettant de faire face à un problème ou un besoin précis. Mais appliqué à une société, ce mot désignait un mouvement général vers la réalisation de valeurs positives définies : dans l'univers des valeurs bourgeoises qu'annonçait la Révolution, il s'agissait surtout de celles du confort et du productivisme, - de l'efficacité et de l'économie de moyens. Cette maîtrise des moyens et de la compréhension du monde qui en découle sont de nature cumulative. L'affrontement entre cet idéal et l'appauvrissement réel des couches laborieuses pendant l'ère de l'industrialisation décrite par Ricardo allait inciter certains à lier ce concept à une teneur morale ; il s'agissait d'améliorer les conditions de vie d'une classe productive atteinte par des fléaux moraux ${ }^{16}$.

8 La projection du devenir de la nature décrite par les sciences dures conduira au projet de cerner l'évolution d'un corps social en tant qu'unité. Cette démarche n'allait pas résister à une analyse néokantienne, représentant une régression épistémologique par rapport à la sociologie historique et compréhensive de Montesquieu ${ }^{17}$. Mais l'œuvre d'Auguste Comte représente une étape décisive dans le développement des sciences sociales en Europe, et ses ambitions ont dominé ce champ pendant le reste du dixneuvième siècle. La tâche explicite de certains sociologues allemands du vingtième siècle était de libérer leur discipline des carcans méthodologiques et idéologiques qu'elle avait hérités du positivisme. Le joug principal était celui des concepts tels que "progrès ", comme le rappelle le titre d'un ouvrage de l'économiste F. von GottlOttilienfeld, Die Herrschaft des Wortes. Dans son Opuscule de philosophie sociale, Auguste Comte avait entrepris une "sommaire appréciation de l'ensemble du passé moderne »: Le progrès dans l'accumulation du savoir permettait aux «savants » de se substituer à la hiérocratie au moment même où les "industriels» se substituaient aux guerriers. Comte propose " un prospectus des travaux nécessaires pour réorganiser la société ». La loi des trois étapes développée ensuite dans son Cours de philosophie positive est une loi de progrès, et la religion laïque qui devait en résulter pour former le consensus social est une religion du progrès humain vers l'excellence. La science sert, lors du passage d'une étape de la conscience à la suivante, de sage-femme, et une technocratie aura la tâche d'atténuer les souffrances qui accompagnent toute transformation sociale. L'évolution est en soi inéluctable, même s'il existe, entre les peuples, des décalages, que Comte réduira à des différences de race.

C'est avant tout en prenant ses distances par rapport aux valeurs progressistes de ce modèle que la sociologie moderne allemande se définira. Certes, le premier à développer cette dialectique fut Auguste Comte, et la crédibilité de son projet intellectuel allait souffrir de sa conclusion ultérieure selon laquelle la spiritualité africaine fut supérieure à celle des bourgeois de l'Europe ${ }^{18}$. Si cette reconnaissance de l'hétérogénéité des valeurs et de la possibilité de concevoir des progrès évidents comme symptômes d'un processus de déclin marquait la fin de la sociologie d'Auguste Comte, elle constituait, avec la publication de Gemeinschaft und Gesellschaft de Ferdinand Tönnies en 1887, le début de la sociologie moderne allemande. La mise en cause de l'idéologie du progrès imprégnera la sociologie allemande du vingtième siècle, influencée de "façon omniprésente... et souterraine $~^{19}$ par Gemeinschaft und Gesellschaft et s'opposera à la légitimation des pouvoirs laïques d'une société en voie de modernisation, attitude typique de la sociologie française, même jusqu'à la troisième République. Durkheim rejette, dans l'une des premières critiques de l'ouvrage de Tönnies, la lecture du devenir de la société moderne comme étant la mécanisation des communautés organiques de jadis, proposant, au contraire, d'y voir une évolution de structures mécaniques et simples vers des structures organiques et complexes ${ }^{20}$. La 
réception frileuse, voire hostile de la sociologie allemande en France décrite par Raymond Aron $^{21}$ témoigne d'une distance et d'un scepticisme tant idéologique que méthodologique.

10 Les attaques des philosophes de l'Histoire, comme Wilhelm Dilthey, Georg Simmel et Heinrich Rickert contre les méthodes de la sociologie positiviste, rappelant celles de l'École historique allemande contre le Droit naturel, accompagnèrent le refus de plus en plus répandu en Allemagne des valeurs liées à l'idée de progrès. Le scepticisme à l'égard de la notion de progrès devient général. L'industrialisation a mis en évidence, aux côtés du progrès, des phénomènes de décadence, et le " pessimisme » atteint, audelà de la contemplation esthétique et blasée des membres du mouvement décadent, une population croissante en Allemagne. " Il n'existe pas de doute », selon le verdict du philosophe Wilhelm Windelband dans son discours Pessismismus und Wissenschaft, que

le pessimisme a gagné une place croissante dans les tendances de l'évolution intellectuelle et spirituelle générale qui déterminent et dominent, comme " pouvoirs supérieurs », l'imaginaire et la formation du caractère des individus. Que l'on lutte contre le pessimisme ou qu'on le partage, qu'on le vilipende ou qu'on le loue, qu'on le considère comme folie ou qu'on en fasse une religion, il s'agit d'un fait qu'il faut prendre en compte. Le pessimisme est sans doute l'une des modes les plus répandue, tout au moins à l'intérieur des frontières de la vie culturelle allemande ${ }^{22}$.

11 Windelband attribue ce phénomène «de mode» d'une part à l'affectation aristocratique d'une bourgeoisie de parvenus, d'autre part aux agitations des socialistes. Il existait

des cercles sociaux entiers, où l'on est mal considéré si l'on se déclare satisfait de l'état du monde, et où l'on supporte la misère de l'existence avec un sentiment partagé de condescendance et avec une expression compatissante de résignation. Il n'existe rien au monde qui flatte davantage la vanité de l'individu que d'afficher sa supériorité non seulement par rapport à tout individu, mais par rapport à l'existence et à la vie tout court!... Mais le pessimisme n'est pas seulement une affaire d'aristocrates: on le prêche autant dans les rues que dans les salons, il accompagne les agitations créatrices de nouveaux besoins dans les cercles laborieux, donnant aux travailleurs une conscience de l'insuffisance de leur situation réelle, il s'infiltre ainsi, comme morosité et incommodité, dans toutes les couches de la population ${ }^{23}$.

\section{La réévaluation du progrès dans les sciences humaines}

C'est la promesse du progrès qui génère la conscience du déclin. Georg Simmel allait développer, dans son essai Sur la question fondamentale du pessimisme du point de vue méthodologique, l'idée que le bonheur ne peut être défini que par rapport au malheur et que toute appréciation d'une prépondérance réelle de l'un ou de l'autre ne pouvait être que parfaitement arbitraire ${ }^{24}$. D'autres encore estimaient que la notion selon laquelle le progrès était une source d'optimisme relevait d'une considération parfaitement creuse de la psychologie humaine. Les derniers Hommes, avec leur croyance au progrès, étaient dominés par des soucis de confort d'une part, par l'efficacité avec laquelle il arrive à devenir instrument d'autre part : fallait-il y voir du progrès? s'était interrogé Nietzsche. Pour l'économiste et le sociologue Werner Sombart, dans son opposition au système de valeurs occidentales lors de la Première Guerre mondiale, c'était le " 
Komfortismus ", selon le néologisme de Sombart, la recherche du confort, qui guidait les actions des « marchands » britanniques, à la différence des « héros » allemands, prêts à défendre des idéaux ${ }^{25}$.

Si la perfectibilité suffit encore pour remplir une vie, la perfection atteinte rendrait cette vie absurde: conclusion qui avait provoqué la crise de sens et la dépression nerveuse célèbre du plus génial apologiste de l'idée de progrès, John Stuart Mill ${ }^{26}$. L'idée que le progrès n'est pas toujours apte à générer du bonheur, mais qu'il peut priver l'individu moderne de toute satisfaction durable a été développée par Max Weber, - écrivant que l'Homme de culture moderne meurt «lebensmüde » et non " lebensgesättigt », las, mais non rassasié de la vie - lorsqu'il distingue entre deux activités dans lesquelles la passion (Leidenschaft) est canalisée par la discipline (Beherrschung), l'art et la science. Ici, c'est le progrès qui prive le scientifique d'immortalité.

Au-delà de ces conditions communes du travail du scientifique et de l'artiste, la science est livrée à un destin qui la distingue foncièrement du travail artistique. Le travail scientifique s'inscrit dans un processus de progrès. Il n'existe pas, dans le domaine de l'art, de progrès dans ce sens. On ne peut pas dire qu'une œuvre d'art d'une époque à laquelle on avait acquis de nouveaux moyens techniques ou une maîtrise des lois de la perspective témoigne a priori d'une supériorité artistique par rapport à une œuvre dont l'artiste ne disposait pas de ces connaissances techniques ou de cette maîtrise des lois, pourvu que l'œuvre rende justice aux matériaux et aux formes, c'est-à-dire si l'objet a été choisi et formé de sorte que d'autres conditions et moyens ne soient pas nécessaires. Une œuvre d'art qui est réellement un " accomplissement" (Erfüllung) ne sera jamais surpassée, ni obsolète; chaque individu peut évaluer sa place à sa façon, mais personne ne prétendrait qu'une œuvre achevée pouvait être surpassée par une autre qui soit également achevée. Tous ceux qui travaillent dans les sciences savent que le produit de leur travail sera obsolète dans les 10,20 ou 50 ans à venir. Voilà le sort, voire le sens du travail scientifique... ${ }^{27}$

L'idée de progrès comme processus cumulatif du savoir ne revalorise pas l'individu, mais participe, au contraire, à la relativisation de sa contribution transcendantale au patrimoine de son espèce. «Le progrès scientifique est une partie, la partie la plus importante, du processus d'intellectualisation auquel nous sommes, depuis des millénaires, assujettis, et par rapport auquel on adopte, de nos jours, habituellement des positions extrêmement négatives ", écrit Weber. L'accumulation du savoir dans une société n'entraîne pas une meilleure compréhension individuelle des réalités qui nous entourent, les « connaissances générales (qu'a l'Homme moderne) des conditions de sa vie " étant sensiblement inférieures à celles dont dispose le sauvage de son environnement. Le progrès scientifique a simplement permis à l'Homme de croire à sa capacité de saisir, de manière rationnelle, les régularités qui régissent les conditions de sa vie. La maîtrise du monde dont se félicite l'Homme du moderne n'est donc que la conviction de pouvoir connaître les règles d'un phénomène, s'il le veut, afin de pouvoir procéder à un calcul de probabilités. Seulement, cette maîtrise signifie «le désenchantement du monde ${ }^{28}$.

Max Weber impose aux sciences humaines une conscience des positions de valeur implicites dans les termes du discours des sciences humaines. "Omettre de former des concepts clairs et nets est particulièrement dangereux dans les discussions pratiques de politique économique et sociale.» L'emploi naïf, dans les sciences économiques, de termes tels que "valeur» ou "productif», qui ne résisteraient à aucune analyse 
conceptuelle, paraît « incroyable » à l'observateur extérieur ${ }^{29}$. Le chercheur doit rester à même de distinguer entre sa description des faits et ses prises de position ${ }^{30}$.

En employant le mot " progrès " pour désigner une évolution sociétale, on adopte une perspective qui dépasse complètement celle des individus porteurs de cette évolution. Les constructions du progrès entrânent les mêmes risques que celles du développement ou de l'évolution - Entwicklung. Si Weber reconnaît que la construction d'idéaltypes d'évolutions historiques (Entwicklungen), peut être «d'une valeur heuristique considérable", il souligne le "très grand risque de confusion entre l'idéaltype et la réalité31. » Il rappelle " que les constructions idéaltypiques d'évolutions et l'Histoire sont deux choses qu'il faut rigoureusement distinguer ${ }^{32}$." D'une "importance éminente, voire unique ", les "idéaltypes deviennent "dangereux, dès lors qu'on les imagine comme forces ou tendances, etc., qui ont une valeur empirique ou même réelle (c'est à dire, en fait, métaphysique) ${ }^{33}$. " Ici il se réfère à la téléologie (progressiste) de Karl Marx.

Weber intègre dans les sciences de l'Homme le perspectivisme radical de Nietzsche, qui impose aux acteurs des décisions individuelles fondées sur leurs valeurs (Wertedezisionismus). Ces décisions impliquent nécessairement la conscience de l'existence de coûts: la progression d'une valeur positive implique le retrait d'autres valeurs, et ces valeurs ne sont pas toujours négatives. Son explication de la montée du capitalisme, " ce pouvoir le plus fatal de notre vie moderne ", servira ici d'exemple.

Dans Die protestantische Ethik und der Geist des Kapitalismus, Weber remet radicalement en cause l'idée reçue libérale que l'ascendance du capitalisme relèverait du progrès de la liberté et de la rationalité des individus au service de l'épanouissement de l'espèce humaine. Déjà, l'évolution était loin d'être universelle. Même si «nous nous plaisons à nous imaginer ", écrit-il, que les "phénomènes culturels" de l'Occident s'inscrivent "dans le sens d'un développement de signification et de validité universelles " ${ }^{34}$, le capitalisme n'avait émergé qu'en Occident. C'est cet enchaînement de circonstances uniques, ce "So-und-nicht-anders-Gewordensein» que la science avait à rendre compréhensible. Weber attribue la montée du capitalisme non à la victoire de l'eudémonisme et du calcul rationnel de jouissances supérieures ou ultérieures qui incitent les individus à accumuler des richesses, mais, au contraire, à une conduite de vie ascétique propice à la constitution de l'épargne et donc du capital. L'individu, dans les sociétés où le capitalisme industriel progressa le plus rapidement, ne connaissait pas des mœurs plus libres que ses contemporains. Les fondateurs du capitalisme moderne vivaient dans des sociétés où les doctrines et les valeurs calvinistes imposaient un contrôle social supérieur à celui des croyances qu'ils avaient abandonnées ; voilà le motif pour lequel ils se vouent autant, d'après Weber, au travail, voilà aussi pourquoi ils s'interdisent toute jouissance «sans inhibition » du produit de leurs efforts. Cette explication de l'accumulation originale derrière le capitalisme représente une mise en cause non seulement des présupposés libéraux favorables au capitalisme, mais également du pathos moralisateur socialiste qui lui est hostile. L'appât du gain, moteur du progrès chez Smith, source d'exploitation et d'aliénation chez Marx, ne joue, dans cette théorie, qu'un rôle subordonné dans la genèse du capitalisme. La richesse d'une société - qui n'est que le résultat consolidé de ce processus - apparaît moins comme l'objectif que comme l'incidence de l'activité économique. Pour parler ici du progrès, il faut adopter une position entièrement extérieure à la perspective de ceux qui l'ont généré. Ce perspectivisme wéberien 
permet une critique des doctrines du capitalisme bien plus radicale encore que celle du marxisme. Le progrès économique est présenté comme résultat d'une irrationalité religieuse, ne correspondant à aucune fin cohérente ${ }^{35}$, et la société hédoniste issue de ce capitalisme doit apparaitre, aux yeux de ceux qui l'ont engendrée, comme une déchéance.

Non seulement les origines du progrès économique, mais aussi l'expression «scientifique » de l'idée de progrès seront interprétées comme émanant de croyances religieuses. L'économiste ordolibéral Alexander Rüstow, réfugié à Istanbul pendant le régime nazi, présentera le développement des croyances libérales à l'harmonie des mécanismes du marché comme le résultat d'un « mysticisme » :

La prépondérance des sciences économiques dans le système du libéralisme dans son ensemble était d'abord une conséquence de l'évolution de la pensée, et surtout de la science : ce fut, en effet, dans la théorie économique que le libéralisme avait fait des découvertes qui marquèrent l'époque. Ces découvertes sont l'automatisme de l'économie du marché, l'autorégulation du système de compétition avec son mécanisme d'offre et de demande, et l'harmonie qui se crée, de façon spontanée, entre l'égoïsme de l'individu et le bien-être de tous. Si ce fut le mérite d'Adam Smith de découvrir le premier cette loi économique, toujours est-il que l'idée générale d'une telle harmonie invisible remonte à un passé lointain. Nous retrouvons, dans l'histoire de la philosophie occidentale, l'idée d'une harmonie invisible d'abord dans la cosmologie de Pythagore et dans la philosophie d'Héraclite d'Ephèse, où elle s'applique déjà aux domaines politique et social (...) Pour Héraclite, le logos divin, la raison du monde, pénètre et guide tout, du devenir de l'Univers jusqu'aux actions des individus. (...) La doctrine de l'harmonie divine a été adoptée par les physiocrates. Il n'y a pas de doute que la circulation économique de leur ordre naturel, qu'ils s'imaginent comme merveille de la perfection et de la sagesse, remonte aux Stoïques et finalement à Héraclite. Nous retrouvons, en même temps, une deuxième tendance intellectuelle dans les doctrines des physiocrates. Il s'agit du taoïsme chinois... La doctrine d'Adam Smith de l'autorégulation de l'économie de marché, désormais pilier du libéralisme moderne, est l'achèvement de l'idée physiocrate de l'ordre naturel. La « main invisible » d'Adam Smith contient clairement un élément du mysticisme pythagoricien, et l'harmonie bienfaisante qui guide cette main invisible n'est autre chose que le logos d'Héraclite et des Stoïques, mêlé au tao de Laotse, mais traduit, par la suite, dans le langage anthropomorphique chrétien du déisme ${ }^{36}$.

Différentes démarches de compréhension explicative (erklärendes Verstehen) historique sont mises en œuvre pour remettre en cause en Allemagne la valeur de l'idée libérale de progrès, et ces démarches sont le fait des libéraux. Or, l'idée semble fondatrice du consensus social de l'Europe occidentale. Après les événements de 1945, c'est cette remise en cause allemande qui sera interprétée comme un irrationalisme et qui fera l'objet d'explications historiques. La remise en cause fut générale, et même pour certains de ses participants, injuste ${ }^{37}$. Le modèle explicatif le plus souvent déployé dans le procès d'intention contre les détracteurs allemands du progrès fut, lui aussi, largement débattu en Allemagne : il s'agit du concept de "ressentiment ", développé par Nietzsche et par Scheler ${ }^{38}$. 


\section{La négation du progrès et la manipulation de la mémoire}

21 La critique du progrès en Allemagne occupe une place primordiale dans l'ouvrage Die verspätete Nation de Helmut Plessner, conçu entre 1934 et 1935 et publié en 1959 comme " contribution à l'Histoire du nationalisme allemand ${ }^{39}$. Dans sa discussion de ce qui est « allemand », Plessner se borne à la « kleindeutsche Lösung » de Bismarck, c'est-à-dire excluant à la fois l'empire catholique autrichien et l'expérience suisse allemande. L'ouvrage, qui témoigne d'une érudition, d'une subtilité et d'un courage civil admirables, puise en même temps dans une tradition méthodologique d'essentialisme dans laquelle l'idée diffuse d'une Volksseele joue encore un rôle, - comme le démontre dès le début du livre la mise en exergue d'une citation de l'essai de Thomas Mann, Deutschland und die Deutschen, de 1945 :

Eines mag diese Geschichte (der deutschen Innerlichkeit) zu Gemüte führen : daß es nicht zwei Deutschland gibt, ein böses und ein gutes, sondern nur eines, dem sein Bestes durch Teufelslist zum Bösen ausschlug ${ }^{40}$.

Le désir qu'exprime le philosophe et sociologue, de retour de son exil aux Pays-Bas après l'effondrement de l'Allemagne nazie, est de «sauver ce qui est le meilleur du patrimoine intellectuel et spirituel allemand", patrimoine que les Allemands ne pourraient abandonner qu'en acceptant de «se sacrifier eux-mêmes ${ }^{41}$. " Il ne fallait pas juger l'ensemble des forces politiques, religieuses et sociales en Allemagne sans considération de leur participation ou de leur non-participation à la «situation de 1933 », comme si le nazisme n'avait été que l'expression «d'énergies sous tension dans un réservoir historique, de prédispositions du caractère allemand ». Mais les idées dont Plessner retrace les itinéraires ne sont plus de simples «figures sonores de la situation d'excitation de l'époque »: elles puisent "dans des influences, des traditions et des

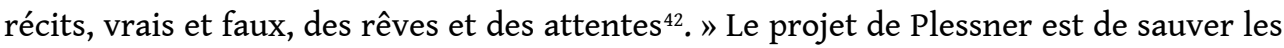
traditions intellectuelles allemandes assimilables à la «communauté de valeurs " de l'Europe occidentale, en évitant à la fois le piège de la mise en place d'une superstructure idéologique sur le chantier de la reconstruction matérielle, telle qu'il l'observe en l'Allemagne de l'Ouest, et celui de l'adultération de l'Histoire pratiquée par les «matérialistes historiques » de l'Allemagne de l'Est, soucieux de reconstruire le devenir du national-socialisme comme l'issue logique de tout ordre économique libéral.

Le Sonderweg allemand s'explique chez Plessner d'abord par l'absence d'un ensemble de facteurs qui avaient été fondateurs dans d'autres sociétés civiles en Europe : l'absence d'une bourgeoisie urbaine, qui, inspirée par les précurseurs des Lumières, pouvait envisager des aspirations individuelles dans un cadre institutionnel suffisamment ouvert pour lui permettre de les développer; l'absence d'une unité politique permettant une réelle mobilité des citoyens ; l'absence d'une Histoire nationale, d'un " âge d'or ", comparable à ceux des pays du "vieil Occident »; l'impuissance dans le concert des nations au dix-neuvième siècle jusqu'à la fondation de l'Empire. Cela a provoqué en Allemagne, après la signature du Traité de Versailles,

un ressentiment national de la disproportion entre sa défaite et sa chute subite

d'une position de grand pouvoir d'une part et le rôle qu'elle avait joué au Moyen

Âge de l'autre, rôle auquel elle n'avait jamais, dans son for intérieur, renoncé.

La montée du nationalisme en Allemagne coïncide avec le rejet de Napoléon, qui venait incarner à la fois la domination étrangère et la volonté d'imposer un progrès 
rationaliste ${ }^{43}$. Dans l'imaginaire des Allemands du dix-neuvième siècle, c'est l'ère du Saint Empire Romain qui occupe, selon Plessner, la place de l'âge d'or. En l'absence de véritables traditions civiles dans une société plus attachée à un esthétisme aristocratique qu'aux valeurs bourgeoises, les pratiques sociales en Allemagne trouvaient leur justification dans l'Histoire :

Voilà pourquoi la problématique de l'Histoire et la critique du progrès n'ont été développées dans aucun des autres pays européens avec une cohérence aussi redoutable qu'en Allemagne ${ }^{44}$.

La modernité associée à la France - sous les formes successives de l'État de Louis XIV, des Lumières, de la Révolution, de Napoléon ${ }^{45}$ - est perçue, selon Plessner, comme étant la menace principale dirigée contre les valeurs anachroniques des Allemands. L'éthique neutre de la démocratie parlementaire, favorisée en France par l'émancipation de l'État laïque par rapport à l'Église et en Grande-Bretagne par le rôle que joue le nonconformisme religieux dans la société, ne trouve pas la même résonance en Allemagne ${ }^{46}$. Si la prise de conscience de la pluralité de confessions en Grande-Bretagne y avait favorisé le libéralisme, le rôle qu'a joué, depuis la Paix de Westphalie, la Staatskirche en Allemagne représentait un obstacle au libre jeu des forces religieuses ${ }^{47}$. En l'absence d'un débat libre comme le débat théologique en Grande-Bretagne ou le débat philosophique en France, les Allemands développent ce que Plessner qualifie tantôt de "weltanschaulicher Ernst ", tantôt de «Weltfrömmigkeit " ${ }^{48}$. Ce sérieux presque religieux dans la réflexion et le déracinement par rapport aux traditions favorise l'émergence de penseurs aussi révolutionnaires et dangereux que Kant, Hegel, Marx ou Nietzsche ${ }^{49}$.

26 Après la Première Guerre mondiale, le scepticisme à l'égard de l'ordre capitaliste et de la politique impérialiste est attisé en Allemagne par le chômage, la propagande de guerre de l'Entente, le Traité de Versailles, les réparations punitives et le jeu des coalitions au sein du parlement de la République de Weimar, entraînant la « dévalorisation totale » des « idées de liberté, de démocratie, d'autodétermination des peuples, de progrès et de paix mondiale, en d'autres termes du système de valeurs de l'humanisme occidental ${ }^{50}$. " Si, pendant cette époque de "désorientation» et de "sensibilité intellectuelle aiguë ", "relativement peu d'Allemands lisaient les œuvres de Spengler et de Klages, de Heidegger et de Carl Schmitt, l'aura de leurs idées a imprégné ce climat d'incertitudes allemandes ${ }^{51}$. »

27 L'industrialisation rapide, l'adoption allemande du modèle américain de production ne s'accompagnèrent pas d'un individualisme de type américain mais d'un renforcement de la tradition de hiérarchies bureaucratiques et militaires ${ }^{52}$. La rigidité des structures et la polarisation des groupes sociaux dans la nation unie ont fait obstacle à la mobilité sociale nécessaire à la réalisation d'un réel projet de société civile, et l'aspect «parvenu» du nouvel Empire et de sa population a généré un désir de noyer le caractère «nouveau riche» dans des traditions réinventées, dans un « Verlegenheitshistorismus ${ }^{53}$, un "Historisme d'embarras» destiné à compenser l'insuffisance de pratiques civiques en Allemagne par les fictions romantiques de la Restauration ${ }^{54}$.

28 Après le refoulement des valeurs de l'humanisme par la contre-révolution et de l'idée de progrès par celle de décadence, la philosophie a finalement été refoulée, au cours de cette modernité allemande, par le matérialisme économique et le matérialisme biologique. Au xixe siècle, la conscience de l'athéisme devient générale, au vingtième, 
c'est la croyance en l'Homme, en l'Humanisme qui meurt, selon Plessner, dans la conscience publique. Les nations hautement civilisées qui assistent à ces crépuscules cherchent alors leurs repères dans un autoritarisme politique, afin de se protéger des conséquences nihilistes et défaitistes qui résultent de ces prises de conscience ${ }^{55}$.

Comme la notion chrétienne de salut cède, selon Plessner, à l'idée du "Weltgericht ", l'hégélianisme est, à son tour, refoulé par la croyance au progrès des positivistes et des saint-simoniens. Cette téléologie, qui permettait à l'individu de la société industrielle de retrouver - dans une progression unilinéaire de faisabilités réalisées - une certaine assurance, est ensuite remise en cause par l'affirmation de la relativité de toute configuration historique ; Ranke exprime ce relativisme, lorsqu'il écrit : «jede Epoche ist unmittelbar zu Gott ». En mal de repères et face à ce relativisme, l'Homme remplit le vide laissé par la religion de jadis par la doctrine matérialiste mais eschatologique de l'évolution biologique, doctrine qui, sous forme vulgarisée, justifiera une croyance à la destinée de la race. La croyance à l'ethnos se politisera, selon Plessner, davantage en Allemagne que dans d'autres nations de l'Europe où le demos avait été plus impliqué dans la mise en place des institutions qui encadraient ses aspirations. Le progrès de l'Homme se réduit, pour un nombre croissant d'Allemands, à l'expression de la prédisposition de son arbre généalogique préhistorique ${ }^{56}$. De fausses mémoires ont été inventées pour mobiliser la "jeune nation", mémoires d'abord d'une histoire mythique, ensuite d'une préhistoire fictive, permettant, lors du Troisième Reich, la destruction des institutions de la société civile qui avaient été créées dans la croyance en un progrès de l'humanité. C'était là une foi que l'Allemagne, selon la lecture de Plessner, ne partageait pas.

\section{Progrès et mémoire dans la pensée de Tönnies}

Les travaux consacrés en Allemagne aux sciences humaines ont conduit à l'élaboration d'un certain nombre d'outils et d'hypothèses permettant une réévaluation de l'idée de progrès. Si cette réévaluation figure chez Plessner comme un facteur parmi d'autres dans l'effondrement de la démocratie et de l'Etat de droit en Allemagne, il ne discrédite pas ces outils ou ces hypothèses en tant que tels, et à la différence de Georg Lukács, n'inculpe pas les sociologues allemands, tous confondus, d'avoir participé à la "destruction de la raison », restant, au contraire, fidèle au principe de considérer la participation ou la non-participation de ces auteurs à la «situation de 1933 ». Les « influences, traditions et récits » des premiers sociologues allemands s'inscrivent, en revanche, dans l'histoire culturelle politisée circonscrite par Helmuth Plessner. Je propose ici de relever les influences et les récits du fondateur de la sociologie moderne allemande, Ferdinand Tönnies, connu à juste titre pour sa position critique à l'égard du " progrès » et pour avoir eu une « influence sur la sociologie allemande contemporaine tellement généralisée, que l'on pouvait croire qu'elle avançait de manière... souterraine $»^{57}$.

Gemeinschaft und Gesellschaft, de Ferdinand Tönnies, a aussi été, à partir de sa deuxième édition, l'ouvrage le plus influent auprès de la Jugendbewegung, le mouvement de la jeunesse allemande. C'est dans cet ouvrage qu'apparaît l'opposition paradigmatique de la sociologie allemande entre communauté et société, c'est dans cet ouvrage que le mot Gemeinschaft est imprégné du pathos qui rendait sa récupération pour les idéologies 
conservatrices et d'extrême droite intéressante, et c'est là que Tönnies présente son propre refus trouble de la bürgerliche Gesellschaft ${ }^{58}$.

La position de Tönnies sur l'idée de « progrès » est des plus ambivalentes. L'évolution moderne représente pour Tönnies à partir des années 1880 jusqu'aux années trente «à la fois un progrès et un déclin ». Tönnies considère, de façon remarquablement explicite, l'ensemble de cette évolution - «die ganze Entwicklung» - comme étant simultanément en progrès et en déclin constant» (« in beständigem Fortgange zugleich und Untergange begriffen $")^{59}$. Dans cet amalgame, il n'y a pas de distinction claire entre les valeurs ascendantes qu'il estime positives et celles qu'il juge négatives, mais le pathos du langage qui traverse l'œuvre permet d'en dégager une idée. Dans une lettre envoyée au philosophe Paulsen pendant la gestation de son ouvrage, Tönnies reconnaît que sa pensée est romantique, et que c'est dans le Moyen Âge qu'il retrouve "l'âge d'or " de son peuple ${ }^{60}$. La théorie qu'il développe est ainsi un diagnostic de l'intellectualisation et de la rationalisation progressives de la société, et avant tout une théorie volitive de l'aliénation progressive des Hommes par rapport à leur nature. Le Kürwille, un type - Tönnies allait le qualifier plus tard de Normalbegriff - de "volonté " qui désigne à la fois le libre arbitre et la raison instrumentale et calculatrice qui domine dans la société moderne, refoule progressivement le Wesenwille, la volonté essentielle, organique, typique des "communautés " d'antan. Si la volonté essentielle - notion reprise plus tard par Heidegger - est alimentée par la mémoire (Gedächtnis) de l'appartenance organique à un être ensemble qui relie ses membres, le Kürwille, qui focalise les individus sur des objectifs matériels projetés dans l'avenir, les divise.

La notion de la mémoire joue ainsi un rôle clef dans une revalorisation de toute valeur matérielle qui précède le " progrès ». La volonté essentielle est enracinée dans le plaisir, le fait de s'habituer, et la mémoire (Gefallen, Gewöhnung, Gedächtnis).

die individuellen Dispositionen und Fähigkeiten (sind) ... höchst verschieden und mit der gesamten Konstitution des Leibes und Geistes, wie sie durch alle Erlebnisse und Erfahrungen hindurch sich ausgebildet hat, verwachsen, weil daraus hervorgegangen ${ }^{61}$.

«Les occupations habituelles qui incombent (à l'individu), donc ses tâches et ses devoirs, deviennent le centre de ses activités mentales. La mémoire (Gedächtnis) est donc une forme de la volonté essentielle, puisqu'elle est le sens du devoir, ... on garde le souvenir de ce que l'on a appris, vécu, pensé comme un trésor ${ }^{62}$. " La mémoire est la source du devoir, de la vénération, et de la création artistique et musicale; elle représente, au-delà des liens avec les vivants, des liens avec les morts eux-mêmes, que l'on continue à connaître et honorer ${ }^{63}$.

La position de l'idée de la mémoire dans l'appareil conceptuel de Tönnies permet de développer une théorie de l'aliénation de l'Homme par rapport à ses propres valeurs ; c'est ici que nous trouvons l'épicentre et la profondeur de la pensée de Tönnies. Si la volonté essentielle valorise les objets qui « plaisent » instinctivement, tout désir d'un objet issu du " progrès » matériel doit paraître chimérique, puisque sans enracinement dans le plaisir instinctif, les habitudes ou la mémoire. Donc sans valeur " essentielle »: on ne peut vouloir des objets nouveaux, inventés d'abord et surtout pour atteindre d'autres fins ultérieures, que de façon "arbitraire ». Cette critique vaut autant pour la production que pour la consommation : si un individu peut, dans le perfectionnement de son art, progresser, la valeur de sa création réside dans les références au passé et dans l'unité de l'acte de la création avec ce passé, ne dépendant pas, d'ailleurs, tant de 
sa perfection que du degré auquel la création même reste en harmonie avec son devenir organique.

Ce rôle que joue la mémoire dans la détermination de ce que l'on veut permet au philosophe normatif qu'était Ferdinand Tönnies de proposer des critères objectifs pour établir la valeur de tout objet pour les individus (toujours en tenant compte de la subjectivité individuelle issue des prédispositions et du vécu). C'est ainsi que le scientifique empirique qu'était Max Weber se voyait obligé de demander à Tönnies, dans leurs échanges au moment de l'institutionnalisation de la sociologie en Allemagne, si son concept de Wesenwille ne contenait pas éventuellement des «jugements de valeur " Le rôle qu'il attribue à la mémoire dans la définition de toute valeur - éthique, esthétique, matérielle - permet à Tönnies de rejeter le "progrès » dans les domaines technologique, industriel et commercial comme principe fondateur de valeurs humaines, en développant, plus profondément que ses prédécesseurs et que ceux qui l'ont suivi, à la fois l'idée marxienne de l'aliénation inhérente au processus de production capitaliste, et l'idée qu'allait promouvoir surtout Galbraith que les besoins $\mathrm{du}$ consommateur moderne sont créés et manipulés à la suite de percées technologiques dans une logique de pur profit.

Dans le domaine de la production, la position de la mémoire chez Tönnies impose la relativisation de tout progrès matériel : le bonheur que poursuit l'Homme moderne lui échappe dès lors qu'il s'engage dans ce processus. Tönnies a la spécificité remarquable, dans ce contexte allemand, de relativiser les croyances progressistes de l'eudémonisme à partir d'une perspective eudémoniste. En dépit des conséquences apparemment conservatrices de cette conception de l'esprit humain, Tönnies ne succombe pas au passéisme, mettant en lumière, au contraire, les libertés de l'esprit qu'entraîne l'évolution moderne dans sa théorie volitive. Tout comme la mémoire, expression de la volonté essentielle (Wesenwille), est à la base de l'art - expression d'une volonté artistique transmise et reçue qui puise dans le passé -, le concept, expression du libre arbitre (Kürwille ), est à la base de la science, apte à définir ses propres lois, dirigée vers l'avenir, et infléchissant l'opinion publique ${ }^{65}$. Certes, la montée de la volonté arbitraire fausse les besoins de l'homme, comme le laisse sous-entendre la citation de Jacob Böhme mise en exergue dans la théorie volitive de Tönnies: «Der Wille ist die Wurzel der Bildnis. Ein falscher Wille zerstört die Bildnis $»^{66}$. Mais elle permet également l'évolution de la conscience et de la liberté de l'esprit.

Tönnies affirme que le processus de rationalisation qu'il décrit représente une évolution linéaire, et qu'il ne pouvait même pas concevoir, avant l'effondrement de la République de Weimar, l'élimination des acquis de la science, de l'État de droit et de la démocratie de la vie moderne; Tönnies est avant tout, comme en témoigne son ouvrage Fortschritt und soziale Entwicklung, publié en 1926, un progressiste mal compris par ses adeptes $^{67}$. Il déclare, dans son autobiographie, que l'on ne pouvait que défendre ce modernisme, en dépit de toute conscience de la tragédie de l'évolution ${ }^{68}$. C'est la tradition du positivisme qui l'immunise contre toute idée de restauration radicale, et la tradition du Droit naturel qui lui sert de repère éthique :

Le vieux souvenir d'un droit communautaire "né avec nous », sommeillant comme un grain de blé dans une momie, susceptible cependant de se développer, s'est maintenu dans l'âme populaire. Compris comme l'idée même de la justice, le droit naturel est, en effet, une possession éternelle et inaliénable de l'esprit humain ${ }^{69}$.

Si certains ont pu lire cet extrait comme expression du désir de la restauration d'une communauté (Gemeinschaft), une lecture précise des textes impose une autre 
conclusion. L'opposition de Tönnies au nazisme n'a pas été une inimitié politique accidentelle; elle est, au contraire, liée à sa théorie et à ses projets intellectuels initiaux. Son «droit communautaire » est avant tout un droit naturel post-libéral destiné à pallier les déficiences du droit sociétaire du libéralisme ${ }^{70}$. Si la mécanisation de tout espace humain représente un appauvrissement de valeurs intérieures, l'individu de la société émergente peut trouver dans une lutte pour la juste répartition des valeurs extérieures qu'entraîne le "progrès » un sens social et une source de renouvellement de la solidarité humaine. Le créateur de ce progrès extérieur est, chez Tönnies, le prolétariat, privé, dans la société capitaliste, de la jouissance des valeurs qu'il a créées. Tönnies s'engage aussi dans le mouvement coopératif, préconisant une démocratie libérale et sociale dotée d'un Etat-providence avec des espaces libres pour l'expression spontanée d'une solidarité sociale. C'est une vision qui s'oppose à tout dirigisme étatique homogène visant une quelconque restauration de la communauté (Gemeinschaft). De telles tentatives marqueraient, selon une prophétie tönniessienne annonçant le totalitarisme, qui paraît remarquable pour un auteur de la fin du dixneuvième siècle, « la fin de la société tout entière et de sa civilisation » ${ }^{71}$.

En lisant l'histoire culturelle politisée de Plessner, il serait facile d'oublier qu'il y avait aussi un progressisme allemand, progressisme qui s'exprime dans le rôle que joue l'Etat wilhelminien dans la promotion de la technologie ou dans le titre de l'organe de la démocratie sociale allemande, Vorwärts ${ }^{72}$. Si Tönnies partageait encore la croyance à un progrès économique et social, sa position reste aussi ambiguë que la collocation " bürgerliche Gesellschaft", une expression qui correspond à la fois à l'idée de la "société bourgeoise » et de la " société civile " ${ }^{73}$, le lexique allemand ne permettant pas d'exprimer une adhésion à la société civile, tout en affirmant des réserves par rapport à la société bourgeoise. La réussite du malentendu de Gemeinschaft und Gesellschaft ${ }^{74}$ est partiellement due à l'hétérogénéité des composants sémantiques de ses concepts.

Certes, d'autres idéologues de la communauté ont désiré, pendant la période weimarienne, substituer aux structures libérales de la République un régime autoritaire. Le fait de libérer le peuple des institutions d'un régime républicain de provenance étrangère et de lui imposer l'adhésion à une communauté raciale sous des auspices vitalistes pouvait signifier pour certains la libération du joug de la responsabilité des individus, abandonnés par l'Histoire à leur seul libre arbitre ${ }^{75}$. Les intentions de Tönnies furent autres. Les critiques de droite de Weimar qui dénoncèrent Tönnies à cause de son adhésion aux doctrines de la tradition libérale et positiviste de l'Europe occidentale avaient davantage raison que les critiques libéraux d'après-guerre qui le dénoncèrent à cause de la récupération nazie de son concept de Gemeinschaft. Son aveu -

Quelques-uns disent que ce fut le succès de ma théorie de la communauté (...) qui inspira l'idéologie nazie, et il y a quelques raisons à cela ${ }^{76}$ ne s'adressait pas à des sociologues ou à des historiens des idées, mais à son fils aîné. At-il éprouvé le besoin, après de longues années d'absence à la suite d'une aliénation précoce, d'exprimer un regret, tout en affirmant, en même temps, le rôle social durable de sa pensée au moment où il est, lui-même, radié de la fonction publique à cause de sa résistance contre la montée du nazisme ${ }^{77}$ ? Certes, Tönnies a légué à la sociologie allemande un regard critique sur les coûts du rationalisme qui domine progressivement tous les champs de la vie moderne. Mais ce processus lui semble en soi inéluctable, et surtout: aucune communauté véritable ne peut être créée en faussant la mémoire. 
Pour Tönnies, le nazisme ne représentait ni un projet de solidarité, ni un retour à la Gemeinschaft, mais une rechute dans la barbarie. Il se sert, dans une déclaration lue à un Congrès de sociologues à Prague en 1935, de la dialectique hégelienne dans une perspective optimiste : le libéralisme ne pouvait être que simplement "aufgehoben », hégelianisme qui suggère que la suppression (Aufhebung) du libéralisme était de nature transitoire, lui permettant de se dépasser (aufheben) dans un retour inéluctable ${ }^{78}$. La création et la manipulation d'une mémoire mythique pour le peuple allemand ne changeant rien à la nature du régime nazi, Tönnies ne se lassa pas de le dénoncer. L'Histoire de la science en Allemagne retient que ce fut l'un des rares sociologues de l'époque à résister.

\section{Progrès et mémoire en allemand}

La notion de progrès s'écrit sous différentes formes en allemand. Elle se traduit, le plus souvent, par le mot "Fortschritt ", mais on la retrouve aussi dans les termes "Fortgang" ou «fortlaufend». La polysémie du préfixe "fort» est plus présente dans ce lexique allemand que dans le mot " progrès »; il désigne à la fois « à l'avant » ou "vers » d'une part et "à partir de» de l'autre. Dans l'évaluation du devenir d'une société, les processus de progrès - dans le sens de «à l'avant» ou "vers» - ne peuvent être constatés que du point de vue des historiens qui décideront, dans un futur lointain, quel sens le tribunal du monde ("Weltgericht») aura attribué aux événéments et aux actions des Hommes. Ici, c'est la fin de l'évolution qui sert de repère pour le jugement, et en ce sens, la force majeure, "die höhere Gewalt », détermine, plus que les Hommes qui agissent, quel "progrès» auront produit, pour un observateur quelconque de l'avenir, leurs actions. La mémoire sélective est définie par les valeurs de cet observateur.

Mais le progrès peut également être conçu dans le sens de «à partir de ». Ici, nous adoptons la perspective des acteurs en amont d'une concaténation de circonstances. Leur souci est de partir d'une situation présente - par la réflexion, la construction, l'invention ou la lutte. Certes, ils connaissent leurs motifs, que l'historien doit s'efforcer de comprendre. Mais ils ignorent l'issue de l'Histoire. Ici, le progrès, le Fortgang n'est autre qu'un «Ausgang » dans le sens de la définition kantienne de "Aufklärung » - «der Ausgang des Menschen aus seiner selbstverschuldeten Unmündigkeit " ${ }^{79}$, le départ d'une tutelle dont on porte une part de responsabilité. L'idée de "selbstverschuldete Unmündigkeit », de la culpabilité de l'Homme par rapport à sa propre sujétion, présuppose, elle aussi, un moment de choix, celui de l'abandon de la liberté.

La mémoire véridique ne suscite pas l'espoir de restaurer un Vécu de jadis, réel ou fictif : comprendre le passé, c'est comprendre qu'il est passé. Elle permet simplement de saisir l'abstraction essentielle : qu'il y a eu, au passé, un état autre que celui du présent, et qu'il est possible, en agissant, de changer - ni plus, ni moins. Ce ne sont ni les sirènes promettant des avancées matérielles, ni les visions concrètes de gloires imaginées d'un passé lointain, qui incitent ceux qui résistent à prendre sur eux d'endurer les périls d'une traversée du désert vers un progrès de l'esprit, vers la liberté : leur destinée est tout autre que certaine. Leur disparition définitive dans les sables ne change en rien la nature progressiste de leur choix. 


\section{NOTES}

1. O. Grauhoff, «Die Germanistik in Frankreich », in : Das gegenwärtige Frankreich, Halberstadt 1926, p. 175 sq, cité in G. Raulet, "Réflexions sur la pratique de "l'Histoire des Idées" », dans : Philosophie et littérature dans les pays de langue allemande au XX ${ }^{e}$ siècle, Germanica, Lille 26/2000, p. 13.

2. Gérard Raulet, op. cit., p. 16.

3. Cette communication a été présentée dans le cadre du groupe de recherche « Histoire des idées dans le monde germanique » (université Lyon 2), à l'occasion d'un séminaire consacré à l'idée de progrès dans l'histoire intellectuelle allemande sous la direction de Fabrice Malkani. Je le remercie de cette occasion de développer ces pensées et de son appui dans la rédaction. Les traductions des sources allemandes sont, en absence d'indications contraires, les miennes. Mes remerciements à Jérôme Blanchard pour une première relecture.

4. Parmi de nombreux exemples, Vor Preussen wird gewarnt, de Christian von Krockow, récemment disparu. Cet article est dédié à sa mémoire.

5. Georg Lukács, Die Zerstörung der Vernunft, Berlin (Ost) 1954.

6. Ernst Fraenkel, Deutschland und die westlichen Demokratien, Kohlhammer, Stuttgart 1979.

7. Wilhelm Hennis, «Zum Problem der deutschen Staatsanschauung», in : Vierteljahreshefte für Zeitgeschichte 7, 1959.

8. Leo Strauss, Natural Right and History, Chicago, 1953.

9. Kurt Sontheimer, Antidemokratisches Denken in der Weimarer Republik, Munich, 1962.

10. L'analyse du processus logique de Verstehen, incontournable pour tout historien des idées, a été développée par Georg Simmel dans Die Probleme der Geschichtsphilosophie, et a trouvé son expression la plus dense dans Wirtschaft und Gesellschaft de Max Weber.

11. Buckle, History of Civilisation in England, London, 1857.

12. En guise d'exemple, l'auteur allemand Hörnigk, suivant Becher, délimite, dans son ouvrage Oesterreich über alles, wenn es nur will de 1684, les biens français comme une catégorie économique, qu'il convenait, à cause de l'effet démoralisant du luxe sur la population, de bannir. Si ces auteurs, comme plus tard von Justi, se caractérisent au sein du mouvement mercantiliste européen par le refus isolé du nouvel ordre moral libéral qu'inaugura le mercantilisme, Hörnigk, comme d'autres après lui, ne peut pas s'empêcher de préconiser très généralement l'adoption des dispositifs productifs de la culture dominante. Klaus von See place la critique allemande des civilisations française et anglo-saxonne dans un contexte plus général d'une auto-revalorisation de valeurs «morales» de la part d'une société matériellement et technologiquement «en retard " par rapport à ses voisins. Klaus von See, Barbar Germane Arier. Die Suche nach der Identität der Deutschen, Universitätsverlag Winter, 1998.

13. Le titre de l'ouvrage de Johann Joachim Becher, "Politischer Discurs von den eigentlichen Ursachen des Auff- und Abnehmens der Städt Länder und Republicken», publié en 1668, est symptomatique : la prospérité relative des entités politiques ou des Etats était l'objet des sciences humaines longtemps avant qu'Adam Smith, sous un titre très semblable, ne s'intéresse à la question de la richesse relative des sociétés.

14. Ferdinand Tönnies, Gemeinschaft und Gesellschaft, Darmstadt, 1979. L'idée qu'il existe une « sociologie allemande » (parallèlement à une sociologie française, britannique, etc.) relève du constat que les contraintes et les circonstances matérielles, institutionnelles et politiques de la production intellectuelle ont défini une discipline propre à cette culture scientifique.

15. Condorcet, Esquisse d'un tableau historique des progrès de l'esprit humain, écrit en 1793, Paris, 1933. Turgot. Tableau philosophique des progrès successifs de l'esprit humain, 1791.

16. L'utilitarisme de Bentham est le lien, dans le contexte britannique, entre les libéraux comme John Stuart Mill et les socialistes comme Robert Owen. 
17. Raymond Aron, Auguste Comte, dans : Les étapes de la pensée sociologique, Gallimard, 1967, p. 77-157.

18. Aron, op. cit.

19. Vierkant, Albert, Gesellschaftslehre, Stuttgart, 1922.

20. Durkheim, Emile, "Gemeinschaft und Gesellschaft », dans : Revue philosophie de la France et de l'Étranger, 27, 1899, pp. 416-422.

21. Raymond Aron, Mémoires, Julliard, 1983, pp. 105, 111, 115.

22. Dans : Wilhelm Windelband, Präludien, II, p. 218. Mohr, Leipzig, 1924, p. 219.

23. Windelband, op. cit., p. $218 f$.

24. Georg Simmel, «Über die Grundfrage des Pessimismus in methodischer Hinsicht ", dans : Zeitschrift für Philosophie und philosophische Kritik, Bd. 90, Falckenberg, Halle-Saale 1887; S. 237-247.

25. Werner Sombart, Händler und Helden, Patriotische Besinnungen, Munich 1915.

26. John Stuart Mill, Autobiography, London, 1873.

27. Max Weber, Wissenschaft als Beruf (ci-après Wissenschaft) In: Gesammelte Aufsätze zur Wissenschaftslehre, hrsg. J. Winckelmann, 7. Aufl., Tübingen, 1988, p 592.

28. Max Weber, op. cit., p. 593, 594.

29. Max Weber, Die 'Objektivität' sozialwissenschaftlicher und sozialpolitischer Erkenntnis (ci-après Objektivität) In: Gesammelte Aufsätze zur Wissenschaftslehre, hrsg. J. Winckelmann, 7. Aufl., Tübingen, 1988, p. 210.

30. Max Weber, Der Sinn der 'Wertfreiheit' der soziologischen und ökonomischen Wissenschaften (ciaprès Wertfreiheit) In: Gesammelte Aufsätze zur Wissenschaftslehre, hrsg. J. Winckelmann, 7. Aufl., Tübingen, 1988, p. 500.

31. Max Weber, Objektivität, p. 203.

32. Max Weber, Objektivität, p. 204.

33. Max Weber, Objektivität, p. 205.

34. Max Weber, Die protestantische Ethik und der Geist des Kapitalismus (ci-après Geist) In: Gesammelte Aufsätze zur Religionssoziologie, 1. Bd., hrsg. J. Winckelmann, 7. Aufl., Tübingen, 1988, p. 1.

35. Une source critique du capitalisme encore peu exploitée me semble se cacher dans la distinction entre "inhaltlicher» et "formeller Rationalismus» qu'opère Weber dans Wirtschaft und Gesellschaft. Max Weber: Gesamtausgabe. - Tübingen: Mohr Siebeck: Abt. 1, Schriften und Reden Bd. 22., Wirtschaft und Gesellschaft: die Wirtschaft und die gesellschaftlichen Ordnungen und Mächte; Nachlaß Teilbd. 5. hrsg. von Wilfried Nippel, Tübingen 1999.

36. Rüstow, Alexander, Die Religion der Marktwirtschaft, Münster : Lit, 2001., p. $19 \mathrm{ff}$.

37. Près de trente ans après la publication de son Problem der deutschen Staatsanschauung, Wilhelm Hennis propose, en guise de réparation, une réécriture de l'Histoire «des Deutschen Geistes ». W. Hennis, Max Webers Fragestellung, Tübingen 1987, p. III.

38. Friedrich Nietzsche, Jenseits von Gut und Böse. In: Sämtliche Werke. Kritische Studienausgabe. Bd. 5. Berlin/ New York, 1980, pp. 7-244. Max Scheler. Das Ressentiment im Aufbau der Moralen, in : Vom Umsturz der Werte, Band 1, 2. Auflage Leipzig 1919.

39. Helmut Plessner, Die Verspätete Nation. Ueber die politische Verführbarkeit bürgerlichen Geistes, Stuttgart, 1959, p. 10.

40. Plessner, op. cit., p. 9.

41. Plessner, op. cit., p. 11.

42. Plessner, op. cit., p. 11.

43. Plessner, op. cit., p. 13.

44. Plessner, op. cit., p. 15f. Pour une discussion des problèmes épistémologiques de la philosophie de l'Histoire allemande voir Catherine Colliot Thélène, Le désenchantement de l'État. De Hegel à Max 
Weber, Les éditions de Minuit, Paris, 1992, et du même auteur : Max Weber et l'Histoire, Paris, P.U.F., 1990.

45. Plessner, op. cit., p. 57.

46. Plessner, op. cit., p. $62 \mathrm{f}$.

47. Plessner, op. cit., p. 66.

48. Plessner, op. cit., p. 69.

49. Plessner, op. cit., p. 82.

50. Plessner, op. cit., p. 39.

51. Plessner, op. cit., p. 17, « incertitudes allemandes » en français dans le texte.

52. Plessner, op. cit., p. $93 \mathrm{f}$

53. Plessner, op. cit., p. 93.

54. Plessner, op. cit., p. 96.

55. Plessner, p. 100 à 101.

56. Plessner, p. 107-118.

57. A. Vierkandt, Gesellschaftslehre, Stuttgart, 1922.

58. Niall Bond, «Le refus de la bürgerliche Gesellschaft et la genèse de la sociologie moderne allemande : l'exemple de Gemeinschaft und Gesellschaft de Ferdinand Tönnies ». Dans : De la société à la sociologie, éd. Catherine Colliot-Thélène et Jean-François Kervégan, ENS-éditions, 2002, p. 117.

59. Tönnies, Gemeinschaft und Gesellschaft, p. 220.

60. Tönnies-Paulsen Briefwechsel. p. 61. Briefwechsel 1876-1908 / Ferdinand Tönnies ; Friedrich Paulsen. Hrsg. von Olaf Klose et al. Kiel : Hirt, 1961.

61. Tönnies, Gemeinschaft und Gesellschaft, p. 102. C'est nous qui soulignons.

62. Ebenda, p. $103 f$.

63. Ebenda, p. 104, p. 136, p. 187.

64. Niall Bond, «Tönnies und Weber», dans : Soziologisches Jahrbuch, Trento, II 1988.

65. Tönnies, Gemeinschaft und Gesellschaft, p. 216.

66. Ebenda, p. 71 .

67. Ferdinand Tönnies. Fortschritt und soziale Entwicklung, Geschichtsphilosophische Ansichten, Karlsruhe 1926. La « réussite » de ce malentendu est très largement commentée par Dirk Käsler dans : «Gemeinschaft und Gesellschaft, Erfolg eines Missverständnisses? » dans : Soziologisches Jahrbuch, Trento, II 1988.

68. Die deutsche Philosophie in Selbstdarstellung.

69. Niall Bond, 2002, p. 115.

70. Ferdinand Tönnies, Die Erneuerung des Naturrechts, 1881. Ouvrage non publié, archives de Ferdinand Tönnies à la bibliothèque de Schleswig-Holstein, Kiel.

71. Ferdinand Gesellschaft, Gemeinschaft und Gesellschaft.

72. Thomas Nipperdey, Deutsche Geschichte 1866-1918. I. Arbeitswelt und Bürgergeist. Munich, 4. Aufl., 1987. Aussi : Christian Graf von Krockow, Die Deutschen in ihrem Jahrhundert 1890-1990, Reinbek bei Hamburg 1990.

73. Niall Bond, «The cross-cultural translation of ideology ». Dans : Parallèles, Cahiers de l'École de Traduction et d'Interprétation, 19, Genève, 1998.

74. Dirk Käsler. op. cit.

75. L'irresponsabilité est ainsi, selon Sebastien Haffner, le principe constitutionnel du régime hitlérien. Anmerkungen zu Hitler, Kindler Verlag, 1978.

76. Traduction de G. Raulet, op. cit., p. 25.

77. Jürgen Zander. «Sieg der Vernunft? Ferdinand Tönnies' Konfrontation mit dem Nationalismus ». In: Landesgeschichte und Landesbibliothek. Studien zur Geschichte und Kultur Schleswig-Holsteins. Hans F. Rothert zum 65. Geburtstag. Hrsg. Dieter Lohmeier und Renate Paczkowski. Heide in Holstein, 2002, p. 171-189.

78. Ferdinand Tönnies, A New Evaluation, Leyden : E. J. Brill, 1973. 
79. Immanuel Kant, in Was ist Aufklärung?: Thesen und Definitionen. Hrsg. von Ehrhard Bahr. Stuttgart: Reclam, 1996. Je remercie le philosophe Walter Oswalt pour son apport à ces réflexions.

\section{RÉSUMÉS}

La montée singulière de l'idée de progrès et son évolution en Europe se décline différemment selon les cultures nationales; mais rares sont les concepts qui induisent autant, de part et d'autre, des comparaisons entre les États-nations, rares aussi des notions autant fondatrices d'identité, porteuses d'espoirs et inspiratrices d'actes. Le retard de l'Allemagne souligné par $\mathrm{H}$. Plessner par rapport aux «évolutions » ou au «développement » que les idéologies dominantes du progrès estiment désirables, est déterminant dans la définition des valeurs et des aspirations dans les pays germanophones. L'un des avantages de l'Histoire des idées allemandes, du caméralisme à l'unification de l'Empire et au-delà, demeure l'optique radicale qu'adoptent, dans l'observation des centres du pouvoir et des institutions dans les pays occidentaux voisins, des auteurs conscients de leur position périphérique. La discussion tantôt logique, tantôt psychologique de Max Weber sur l'application de l'idée de progrès aux productions artistique et scientifique révèle les faiblesses de cette idée dans sa dimension existentielle, et son analyse de la genèse de la forme sociétale dominante, généralement considérée comme l'issue du progrès, présente l'apogée d'une eschatologie progressiste comme simple effet pervers des actions de ses géniteurs. L'anthropologie d'inspiration mystique de Tönnies, qui permet de redéfinir la réussite d'une action selon des critères intérieurs, sert à élaborer une critique des idéologies du succès extérieur. La récupération de la notion de progrès par un certain scepticisme allemand pendant l'époque la plus noire de l'Histoire intellectuelle allemande n'affaiblit en rien le potentiel libérateur des critiques, entre autres libérales, de l'héritage libéral européen exprimées en Allemagne ; elle ne devrait pas l'occulter non plus. Le développement de l'idée de progrès à partir d'expériences allemandes et de la langue allemande entre dans un patrimoine intellectuel plus vaste, patrimoine auquel l'Homme peut puiser, dans la production consciente de sa réalité et de ses perceptions.

Das einzigartige Aufkommen der Fortschrittsidee und ihre Entwicklung im gesamteuropäischen Zusammenhang ist national differenziert zu begreifen; wie kaum ein Begriff führte und verleitete dieser zu Vergleichen zwischen Nationalstaaten, wie kaum eine Idee stiftete diese hoffungstragende und handlungsleitende Idee Identitäten im Verlaufe der Moderne. Ein entscheidendes Moment in der Bestimmung gesellschaftlicher Wertungen und Bestrebungen im deutschsprachigen Raum bildet die von H. Plessner unterstrichene Verspätung Deutschlands in den «Entwicklungen », welche die führenden Fortschrittsideologien des westlichen Europas als Wünschbarkeiten hervorhoben. Somit liegt ein Vorzug deutscher Ideengeschichte schon bei den Kameralisten und über die Reichsgründung hinaus in der periphären und dadurch radikalen Sicht ihrer Träger gegenüber den Machtzentren und den Institutionen der westlichen Nachbaren. Max Webers logische und psychologische Auseinandersetzung mit der Anwendung der Fortschrittsidee auf die Bereiche der Kunst und der Wissenschaft legte ihre Schwäche in der existentiellen Sinngebung bloss, und seine Analyse der Genese der Gesellschaftsform, die man gemeinhin als Ergebnis des Fortschrittes ansah, stellte jene eschatologische Höhe als perversen Effekt dar. Das ideologiekritische Potential der Kupplung vom Erfolgsurteil an Kriterien der 
Innerlichkeit wurde in einer mystisch inspirierten Anthropologie von Ferdinand Tönnies entwickelt. So entkräftigt die Vereinnahmung der Fortschrittsskepsis während der dunkelsten Kapitel der deutschen Ideengeschichte mitnichten das befreiende Potential u.a. liberaler deutscher Kritiken am liberalen Vermächtnis Westeuropas, und sie sollte sie auch nicht überschatten. Die aus deutschen geschichtlichen Erfahrungen und sprachlichen Formen speisende Fortentwicklung dieser Idee gehört zu einem allgemeineren Besitz des Menschen in der bewussten Gestaltung von seiner Wirklichkeit wie von seiner Wahrnehmung.

\section{AUTEUR}

\section{NIALL BOND}

Université Lumière Lyon 2 